ORIGINAL ARTICLE

\title{
STUDIES ON THE DISTRIBUTION OF CLINICALLY DIAGNOSED PULMONARY TUBERCULOSIS IN EBONYI STATE, NIGERIA
}

*Obi, R.K ${ }^{1}$, Amadi, A.N. ${ }^{2}$, Idika I.M ${ }^{3}$, Nwanebu, F.C 1 Departments of Microbiology ${ }^{1}$, and Public Health Technology², Federal University of Technology, Owerri, Imo State and Presbyterian Joint Hospital ${ }^{3}$, Uburu, Ebonyi State, Nigeria.

*Author for correspondence: robertobi_2003@yahoo.ca

Abstract

A study was conducted between January, 2005 to December, 2006 to ascertain the prevalence of pulmonary tuberculosis among patients who attended chest clinics in some randomly selected hospitals, Clinics and Health Centers in Ebonyi State, Nigeria. Investigations were carried out using the two popular diagnostic criteria for pulmonary tuberculosis namely the specific Ziehl Neelsen (ZN) methods and chest X-ray. A total of 962 patients with clinical signs and symptoms of tuberculosis were studied. Out of this figure, $559(58.1 \%)$ had pulmonary tuberculosis with 1:1 male/female ratio. There was no significant difference between $\mathrm{ZN}$ sputum smear positive and chest $\mathrm{x}$-ray in the diagnosis of pulmonary tuberculosis in the studied population at $95 \%$ confidence level. The possible reasons for the high prevalence of pulmonary tuberculosis may be attributed to increase in the incidence of HIV/AIDS, high rate of poverty, emergence of drug-resistant strains of Mycobacteria and to a lesser extent, smoking and diabetes. Key words: Pulmonary tuberculosis, sputum, chest X-ray, Ziehl Neelsen.

\section{INTRODUCTION}

Pulmonary tuberculosis (TB) is a common, deadly infectious disease caused by Mycobacteria, mainly Mycobacterium tuberculosis. Tuberculosis most commonly attacks the lungs but can also affect the central nervous system, the lymphatic system, the circulatory system, the genitourinary system, bones, joints and even the skin.

Other Mycobacteria such as Mycobacterium bovis, Mycobacterium africanum, Mycobacterium canetti and Mycobacterium microti can also cause tuberculosis especially in individuals with defective immune systems [1,2] Tuberculosis can develop after inhaling droplets sprayed into the air from a cough or sneeze by someone infected with M. tuberculosis. The risk of contracting TB however increases with frequency of contact with people who have the disease, with crowded or unsanitary living conditions and with poor nutrition[2]. Others at risk of contracting the disease include intravenous drug abusers, medically under-served and low-income populations, and patients immunocompromised by conditions such as HIV/AIDS, and health care workers serving these high risk patients[3].

Initial infection of TB (known as primary pulmonary tuberculosis) occurs when Mycobacteria reach the pulmonary alveoli and invade and 
replicate within alveolar macrophages [4]. This primary stage of the infection is usually asymptomatic and in most cases patients recover from the infection without further evidence of the disease [5]. However in a few number of cases, especially when the immune system does not successfully contain the primary infection, the latent or asymptomatic TB always progresses to the active case. Symptoms of active cases of TB include chest pain, coughing up blood (hemoptysis) and a productive, prolonged cough for more than three weeks. The symptoms could also be systemic and may include fever, chills, night sweats, appetite loss, weight loss, pallor and often, a tendency to fatigue easily [6]. If untreated, active TB disease kills more than half of its victims [7].

Over one third of the world's population are carriers of the bacterium, with new infections occurring at a rate of one per second[6]. This increase in TB resurgence may be attributable to increase in HIV infection and neglect of TB control programs, poor environment, poor nutrition and the emergence of drug resistant strains of the Mycobacterium[8;9]. As a result of the above factors, which also have worsened treatment efforts, TB has now been declared a global health emergency by the World Health Organization [10].

\section{Materials and methods}

\section{Sampling}

A total of 962 patients with suspected cases of pulmonary tuberculosis who presented to different chest clinics in some randomly selected hospitals and clinics in Ebonyi State were used for this two-year study which spanned from January 2005 to December 2006. This included 648 males and 314 females. The purpose of the study was fully explained to them by the medical officers and their informed consents obtained prior to the study as recommended by the World Health Organization [11].

\section{Diagnosis}

A combination of chest $x$-ray and $\mathrm{ZN}$ techniques was used to screen the patients after physical examinations were made to assess their general health status. Sputum samples were obtained from patients showing signs of prolonged coughing to investigate for the presence of Mycobacterium tuberculosis using the Zieehl-Neelson's acid fast staining method as applied by[12]. In the process each sputum specimen was swirled in its neat screw - capped container and with the help of a wire loop, smeared at the middle of a clean glass slide. The smear was airdried, heat-fixed over the bunsen burner flame and flooded with strong carbol fuchsin solution with the application of gentle heat for five minutes, on a staining rack. The smear 
was next washed with water and decolourized by covering with 3\% acid - alcohol for 10 minutes. It was washed again with water and counter stained using Loeffler's methylene blue solution for 30 seconds. The stained smear was allowed to air-dry and examined microscopically using oil immersion (x1000) objective. The acid - fast bacteria appeared as bright red coloured rods that stood out clearly against the blue background of methylene blue, indicating positive cases. A parallel chest $\mathrm{x}$-ray test was run on the same patients. The chest $x$ ray which involved the standard posterior - anterior view was used as an extra confirmatory test for tuberculosis as recommended by[13]. In the test, cavitary lesions in the upper lobe of the lungs or mediastinal lymphadenopathy accompanied by patchy irregular consolidations indicated active pulmonary $\mathrm{TB}$ cases. Inactive cases were observed as fibrocalcific scar within the lungs.

\section{Results}

The results (Table 1) showed that a total number of 559 (58\%) out of the 962 were diagnosed as PTB in the study. This is the sum of the occurrence in 2005 with 260 cases (27\%) and 2006 with 299 cases $(31 \%)$. There was no significant difference $(p>0.05)$ between the rate of PTB occurrence in years 2005 and 2006. The results as seen in Table 2 also showed that 324 (34\%) males and 235 (24\%) females tested positive for PTB.

TABLE 1: DISTRIBUTION OF PULMONARY TUBERCULOSIS AMONG PATIENTS IN UBURU, EBONYI STATE.

\begin{tabular}{rllll}
\hline YEAR & TOTAL & ACTIVE PTB & INACTIVE PTB & PERCENTAGE ACTIVE PTB \\
& SCREENED & & & \\
\hline 2005 & 461 & 260 & 201 & 27 \\
2006 & 501 & 299 & 202 & 31 \\
\hline TOTAL & 962 & 559 & 403 & 58 \\
\hline
\end{tabular}

TABLE 2: DISTRIBUTION OF PTB AMONGST PATIENTS ACCORDING TO AND SEX IN UBURU, EBONYI STATE.

\begin{tabular}{lll}
\hline YEAR & Males & Female \\
\hline 2005 & 152 & 108 \\
2006 & 172 & 127 \\
\hline TOTAL & $324(34 \%)$ & $235(24 \%)$ \\
\hline
\end{tabular}




\section{Discussion}

In 2005, 260 (27\%) active TB cases were diagnosed in the community under survey. The figure rose to $299(31 \%)$ in 2006. This represents an increase of 39 (4\%) showing that more people may have been exposed to the pre-disposing factors that lead to the observed increase in the incidence of the disease in a space of only one year ( Table 1).

Similarly, 152 (27.2\%) men were diagnosed as active TB cases in 2005 while the number of women was 108 (19.3\%) showing a difference of 44 $(7.9 \%)$ more men than women diagnosed of the infection in the year. In 2006, the number of men diagnosed rose significantly to $172 \quad(30.8 \%)$ representing an increase of 6.2 in the diagnosis of men made in 2005 and 2006, while that of women also increase to $127(22.7 \%)$ similarly representing an increase of $8.1 \%$ in the diagnosis of women in 2005 and 2006. The difference between diagnosis made of women and men was observed to be $45(8.1 \%)$ in the year (Table 2).

Incidence of TB was indeed on a steady increase in Uburu with more men suffering from the ailment than women probably because they were known to indulge more in the pre-disposing factors to the infection than the latter. This observation has been corroborated by that made by the World Health Organization, who reported that the incidence of $\mathrm{TB}$ was on the increase worldwide with nearly 2 billion people, representing one-third of the World's population suffering from the disease 14 . In addition, the Centre for Disease Control and Prevention 15 reported that about 8 million people are added to this figure with about 2 million deaths resulting ailment annually.

Some factors which have been implicated in the re-emergence and subsequent epidemics of TB cases all over the world were also observed to be responsible for the increase in the incidence of the disease in Uburu. These include increase in the incidence of HIV/AIDS; individuals with immune systems damaged by HIV/ AIDS have a higher risk of developing active TB either from new exposure to the disease or a reactivation of dormant Mycobacteria. With the destruction of the immune system, treatment will become more difficult with the result that the disease will become more resistant to therapy[10]. Furthermore, the rate of emergence of drug - resistant strains of Mycobacteria, was enhanced by 
inadequate treatments through not taking the prescribed regimen appropriately, or using low quality medications [16;2]. Drug resistant Mycobacteria caused an increase of about $20 \%$ in TB resistance to standard treatments and $2 \%$ resistant to secondline drugs recorded between 2000 and 2004[5]. In addition there was a high level of poverty observed among the people in Uburu which must have contributed to the high prevalence of $\mathrm{TB}$ in the area. Poverty has been reported to be a very important factor in disease progression and prevalence in developing countries especially SubSaharan Africa where majority of the twenty countries of the world with the highest rates of $\mathrm{TB}$ cases are said to be located[10]. Finally[17] reported that in addition to other factors, smoking more than 20 sticks of cigarette every day could increase the risk of succumbing to TB infections, while[18] identified diabetes mellitus as the greatest predisposing risk factor to this disease that is growing in importance in developing countries. Similar factors may also have been responsible for the rising cases of $\mathrm{TB}$ noticed in Uburu.

With the incidence of HIV/AIDS pandemic which was reported[19] to be highly prevalent in Nigeria still wreaking havoc on the health status of many individuals, the re-emergence of TB epidemics in addition may initiate calls for a declaration of a state of emergency in the health sector. Fortunately, the WHO has already declared the disease a global health emergency and has instituted several measures to assist developing countries to control the infection and spread of Mycobacteria. The Nigerian government on its part is complementing this effort through its adequate provision of preventive BCG vaccines for infant vaccination in various hospitals and health centers; and numerous radio, newspaper, and television advertisements, urging anyone with suspected signs and symptoms of the disease or who is positive for the HIV virus to report for immediate diagnosis for TB. Positive individuals would commence early treatments with antituberculous drugs distributed freely at government-owned hospitals, health centers and clinics.

However, the persistence and upsurge in TB cases today despite this generosity from government, calls for stringent managerial policies to be instituted to handle cases and contain the epidemic before the greater population in this country falls victim to the infection. To this effect, efforts should be made to improve the standard of living of every 
Nigerian through adequate provision of basic and essential amenities such as potable water, good food and affordable housing. Also in any situation where a patient has been diagnosed as a TB case, adequate control measures should be instituted to break the chain of possible transmission of infection to others by isolating such a patient compulsorily in a health center or clinic where intensive care could be given. Antituberculous drugs administered to such a patient should be closely monitored to ensure strict compliance to prescriptions and duration of treatments and thus prevent any form of drug resistance developing as a result of treatment failures. Furthermore, since inactive TB cases could progress to active forms later in life, especially after a reactivating infection of HIV, it will only become reasonable to subject all inactive cases to appropriate treatment with antituberculous drugs. In addition, campaigns on the treatment, prevention and control of HIV/AIDS should be intensified. Government must create adequate awareness on the aetiology and epidemiology of TB infection, stressing the need for healthy living involving good feeding, clean environment and adequate housing. However, government should provide relevant health institutions with diagnostic equipment and materials to detect Mycobacteria. Trained environmental personnel should be posted to the Local Government Areas to monitor public compliance to laiddown policies on control and early detection of any outbreaks of the disease. Finally adequate protection should be given to all medical personnel and healthcare providers who handle TB patients, through immunization, payment of adequate hazard allowances and training since their constant contact with these high risk patients is a danger, not only to themselves but to the larger part of the society.

\section{REFERENCES}

1. Raviglione, M.C and O'Brien, R.J (2004). Tuberculosis in Kasper, D.L, Braunwald, E., Fauci, A.S., Hauser, S.L., Longo, D.L., Jameson, J.L., Isselbacher, K.J. eds: Harrisons Principles of Internal Medicine, $16^{\text {th }}$ ed., McGraw-Hill Professional, 95366.

2. Ferrara, G., Loci M, Meacci, M (2005). Routine Hospital use of a New Commercial Whole Blood Interferon - (gamma) Assay for the Diagnosis of Tuberculosis Infection. Am J. Respir Crit Care Med. 172(5): 6315 
3. Griffith, D., and Kerr, C 91996).

"tuberculosis: disease of the past, disease of the present." $J$.

Perianesth Nurs 11(4): 204-5.

4. Onyebujoh, P., and Rook G.A.W (2004). World Health Organization Disease Watch: Focus: Tuberculosis. http:/ / en:wikipedia.org/wiki/ tuberculosis.

5. Madison, B. (2006). Emergence of Mycobacterium tuberculosis with extensive resistance to second line drugs worldwide, 2000-2004. MMWR Morb Mortal Wkly Rep 55(11): 301-5.

6. World Health Organization (2006). Tuberculosis fact sheet No:14 Global and regional incidence. http://www.who./mediacente r/factsheets/fs14/en/.

7. Sobero, R., Peabody, J. (2006). Tuberculosis control in Bolivia, Chile, Colombia, and Peru: why does the incidence vary so much between neighbours? Int J. Tuberc. Lung Dis 10 (11):12925.

8. Iademarco, M.F., and Castro, K.G. (2003). Epidemiology of tuberculosis. Seminars in respiratory infections 18(4): 22540.

9. CDC (2003) Division of Tuberculosis elimination. Core
Curriculum on tuberculosis: What Clinicians should know. $4^{\text {th }}$ edition. http:// www. Cdc/gov/ncidod/dbmd/disea seinfo/tuberculosi_g.htm.

10. World Health Organization (2006) Global tuberculosis control - surveillance, planning, financing.

http://en.wikipedia.org/wiki/ surveillance_planning_financin $\mathrm{g}$

11. TDR (2002) work look for investigators UNDP / World Bank / WHO Special Programme for Research and Training on Tropical Diseases (TDR / PRD / GCP / 02). Pp30

12. Madison, B (2001). Application of stains in clinical microbiology Biotech Histochem 76(3): 119-25.

13. Nahid, P., Pai, M., Hopewell, P. (2006). Advances in diagnosis and treatment of tuberculosis. Trends Microbiol 6(3):107-12.

14. National Institute of Allergy and Infectious Diseases (NIAID) (2005). Microbes in sickness and in health. http://www.niaid.nih.gov/fact sheets/microb_sickness_health. $\underline{\mathrm{htm}}$.

15. CDC (2006). 2005 Surveillance Slide 
Set.http://www.Cdc/gov/ncid

od/dbmd/diseaseinfo_surveill

ance/tuberculosi_g.htm.

16. O' Brien, R. (1994). Drug resistant tuberculosis: etiology, management and prevention. Semin Respire Infect. 9(2): 104-12.

17. Davies, P.D.O, Yew W.W., Ganguly, D. (2006). “Smoking and tuberculosis: the epidemiological association and pathogenesis." Trans $R$ Soc Trop Med Hyg 100:291-8.
18. Restrepo, b.I. (2007). Convergence of the tuberculosis and diabetes epidemics: renewal of old acquaintances. Clin Infect Dis 45:436-8.

19. Obi, R.K, Iroagba, I.I, Ojiako, O.A, (2007). Prevalence of human immunodeficiency virus (HIV) infection among pregnant women in an antenatal Clinic in Port Harcourt, Nigeria. Afr. J. Biotechnol 6(3): 263-266.

Visit our website: http//www.ajol.info/journals/ajcem 
